\title{
The microbiome of the invertebrate model host Galleria mellonella is dominated by Enterococcus
}

\author{
Camille Nina Allonsius ${ }^{\dagger}$, Wannes Van Beeck ${ }^{\dagger}$, Ilke De Boeck, Stijn Wittouck and Sarah Lebeer
}

\begin{abstract}
Background: The popularity of Galleria mellonella as invertebrate model is increasing rapidly, because it forms an attractive alternative to study bacterial, fungal and viral infections, toxin biology, and to screen antimicrobial drugs. For a number of vertebrate and invertebrate animal and plant models, it has been established that the commensals present within the microbial communities on various host surfaces will influence the host's immune and growth development state and the colonization capacity of newly introduced micro-organisms. The microbial communities of Galleria mellonella larvae have, however, not yet been well characterized.

Results: In this study, we present the bacterial communities that were found by 165 rRNA amplicon sequencing on different body sites of $G$. mellonella larvae. These communities showed very little diversity and were mostly dominated by one Enterococcus taxon. In addition, we found that the production conditions (as 'bait' for fishing or under more controlled 'research grade' conditions - with or without hormones and antibiotics) appear to have little impact on the microbiota of the larvae.
\end{abstract}

Conclusions: Establishment of the simplicity of the microbiota of G. mellonella larvae underlines the potential of the larvae as a model host system for microbiome-host interactions.

Keywords: Galleria mellonella, Greater wax moth, Microbiota, Enterococcus, Invertebrate host, Low diversity

\section{Background}

The invertebrate model Galleria mellonella or greater wax moth has become a popular alternative to rodent models in several research areas, including bacterial and fungal virulence, viral infections, toxin research and antimicrobial drug testing. This is thanks to the lack of ethical constraints, its short life cycle and its simplicity regarding handing and necessary lab equipment [1-3]. Despite the increasing body of literature using G. mellonella larvae, not much is known about their commensal microbiota. To the best of our knowledge, only four studies have used high-throughput $16 S$ rRNA gene sequencing for the characterization of the gut microbiota of the larvae. The focus of these studies was, however,

\footnotetext{
* Correspondence: sarah.lebeer@uantwerpen.be

${ }^{\dagger}$ Camille Nina Allonsius and Wannes Van Beeck contributed equally to this work.

Department of Bioscience Engineering, Research Group Environmental Ecology and Applied Microbiology, University of Antwerp,

Groenenborgerlaan 171, B-2020 Antwerp, Belgium
}

on how G. mellonella manages its gut microbiota during metamorphosis, on the influence of antibiotic feeding on alterations in antibiotic resistance gene pool in the gut microbiota, and the effect of particular Photorhabdus strains and envenomation by the ectoparasitoid Habrobracon hebetor on the gut microbiota [4-7]. The bacterial diversity of different body niches (e.g. gut versus haemolymph) and interindividual variation has not yet been mapped.

The microbial community of the moth might play a major role in the colonization capacity of newly introduced bacterial species and in the immune state and maturation of the larvae. For example, for the model invertebrate Drosophila melanogaster, it has been shown that flies with a normal microbiota, existing of Acetobacter and Lactobacillus species that probably originate from their feed, are less susceptible to certain infections than flies depleted of these predominant members $[8,9]$. In addition, the growth and development rate of $D$. melanogaster is shown to be affected by the microbiota

(c) The Author(s). 2019 Open Access This article is distributed under the terms of the Creative Commons Attribution 4.0 International License (http://creativecommons.org/licenses/by/4.0/), which permits unrestricted use, distribution, and 
$[9,10]$. Also in many other invertebrate and vertebrate animal models, even plants, the microbial community will influence the host's immune state and the colonization capacity of newly introduced micro-organisms. Currently, two different types of larvae of G. mellonella are being used in scientific research, i.e. 'research grade' larvae that are grown without hormones or antibiotics and the cheaper 'bait' or 'pet food' larvae, which are grown for fishery, but the consequences on their microbiota have not yet been described.

Mapping the core microbiota of this model animal could improve our interpretation of disease models and studies on toxin research and antimicrobial drug testing. In this study, we aimed to characterize the microbiome present at different body sites of the G. mellonella larvae, namely the skin surface, in the fat body, the haemolymph and the faeces (to represent the gastrointestinal microbiome) of larvae produced under the most commonly used conditions in microbe-host interaction research, namely bait larvae and researchgrade larvae.

\section{Results}

The microbiome of Galleria mellonella larvae shows low diversity at each body site and is dominated by

\section{Enterococcus}

Microbial DNA was obtained from a skin, a faeces, a fat body and a haemolymph sample, from 12 bait and 12 research-grade larvae. To prevent possible cross-contamination, several precautions were taken as outlined in materials and methods. The amplified V4 regions of the $16 \mathrm{~S}$ rRNA genes of the samples were sequenced by Illumina MiSeq. After denoising the raw sequencing output, the sequenced library consisted out of 6,469,678 reads across 104 samples and 1902 taxa. Before further processing this table, a rigourous quality control was applied as described in Jervis \& Bardy [11] as an additional way to account for possible cross contamination. In short, DNA concentrations were estimated by dividing the number of reads by the volume added to the library. Subsequently the relative abundance of each taxon was correlated with this "estimated" DNA concentration (Spearman correlation), and assessed using a fisher exact test. Taxa that were significantly associated with low-concentration samples (negative kit and PCR controls) were removed (threshold, $\mathrm{p}<\mathrm{e}-5$ ). This led to a reduction of taxa from 1633 to 373 taxa. Next, a manual curation of well-known kit contamination species was performed [12]. This lead to a final dataset containing 5,867,192 reads across 96 samples and 343 taxa which was used for downstream analysis including calculations of the diversity between body sites and the generation of overall bacterial community profiles. Of note, we observed significant differences in the concentrations of total extracted DNA across the different body sites studied for both bait and research grade larvae, suggesting a clear difference in bacterial load in these different sites. In general, the haemolymph fluid had the lowest bacterial load, which could be the result of clearance by the immune cells highly abundant in this anatomic site of the larvae [13]. In addition, we also tested whether the rearing conditions influenced the bacterial load in the gut of the larvae by comparing the bacterial DNA concentrations in the faecal samples with qPCR. This comparison showed that the bacterial load in the bait-grade larvae was approximately 5 , 3 times lower than in the research-grade larvae (Fig. 1, $p$ value $=0.06528$ ), which could be the result of the administration of antibiotics during rearing.

Both the Observed Diversity index, which only took the presence/absence of bacterial taxa into account, and the Inverse Simpson diversity index, which also took the evenness of the microbial community into account, showed that the overall diversity of the different body sites was very low (Fig. 2). The alpha diversity was the highest for the skin samples (Inverse-simpson index = 9.98) and the haemolymph samples (Inverse-simpson index $=4.39$ ) of the bait grade larvae. Other body sites had an index ranging from 1.01 (faeces) to 2.14 (fatbodies). When comparing body sites within the type of origin, only the larval skin samples showed a significantly higher diversity compared to the fat body $(p=0.004)$ and gut $(p=0.001)$ for the bait larvae and an increasing trend compared to the gut $(p=0.056)$ for the research-grade larvae.

Strikingly, we found the same amplicon sequence variant (ASV), belonging to the genus Enterococcus (Fig. 3), in all samples from the different body sites studied here. In all samples, except for the skin and haemolymph of the bait larvae studied, this ASV was also dominant (relative abundance $>50 \%$ ). Based on further classification at the EZBioCloud (formarly known as EZtaxon) database [14], this ASV could be classified as Enterococcus gallinarum/saccharolyticus, a common gut commensal of insect, such as Drosophila [15], humans [16] and animals, such as dogs and gulls [17]. To confirm these sequencing results, samples were also plated out on De Man Rogosa Sharpe (MRS) medium and colonies were picked for identification using $16 \mathrm{~S}$ colony PCR and Sanger sequencing. Enterococcus colonies were amongst the most abundant colonies on plate for each of the different body sites. On the skin of the bait larvae however, the community was mainly dominated by two non-bacterial ASVs, classified as a plant-associated mitochondrion (classified as Streptophyta) and chloroplast (classified as Agrostis sp.) (Fig. 3b). Further investigation of these two genera led to the hypothesis that these two taxa are derived from components of the bedding from the larvae enclosures, in agreement with different rearing conditions of bait larvae coming more into contact 


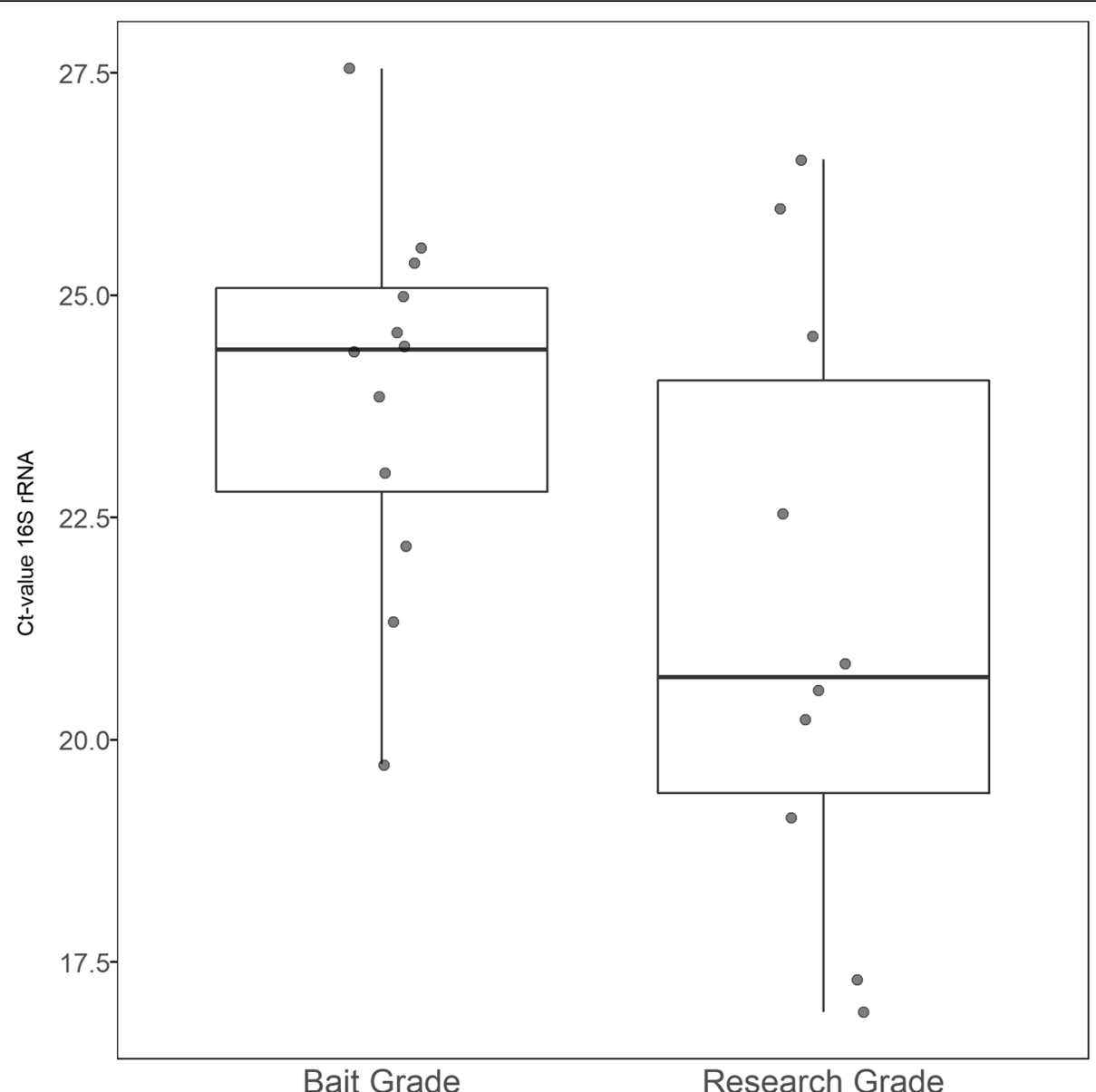

Fig. 1 Comparison of bacterial load in the larvae. The difference in bacterial load between the faecal samples of 12 bait and 12 research-grade larvae was estimated by $\mathrm{qPCR}$ with general primers for the $\mathrm{V} 4$ region of the 165 rRNA gene

with plant material. In addition to the Enterococcus gallinarum/saccharolyticus, a few other lactic acid bacteria were found, including Enterococcus 2 (Enterococcus faecium/faecalis, max $10 \%$ relative abundance), Lactobacillus and Leuconostoc, among other typical gut commensals as Bifidobacterium and Peptoniphilus. Next to these probably commensal bacteria, we also found some potential pathogenic ASVs, mainly in the bait larvae, such as Enterobacter, Pseudomonas, Staphylococcus and Streptococcus.

\section{Discussion}

Our data presented here show that, independent of the body site sampled, G. mellonella larvae used for microbiological research are dominated by a single Enterococcus taxon, putatively identified as E. gallinarum or E. saccharolyticus based on the combination of ASV analysis and the curated EzBioCloud 16S rRNA reference database [18], although various other taxa (such as Staphylococcus, Pseudomonas and Enterobacter species) were also frequently found. The simplicity of the larval microbiota has a number of implications for their role as model host animals. Firstly, as mentioned before, the growth and development rate of model animals are shown to be affected by the microbiota $[9,10]$. Therefore, the robust microbial composition of Galleria mellonella will enhance experimental reproducibility across scientific institutions. Secondly, results of microbiota studies will be easier to interpret, as there will be little variance introduced by differences in the initial microbiota. It should however be noted that despite the presence of only one dominant ASV, there could still be underlying diversity in the form of different very closely related strains within this ASV. It should also be taken into account that the immune system of the larvae might be skewed by the predominant interaction with enterococci. Of note, long term rearing might have promoted the growth of Enterococcus gallinarum/saccharolyticus, abundantly present in the Galleria gut to also colonize the other body sites.

Despite the lack of controlled production of G. mellonella larvae that are sold as bait, but frequently used for experimental setups, the microbial communities in their gut, haemolymph and fat bodies appeared to be dominated 


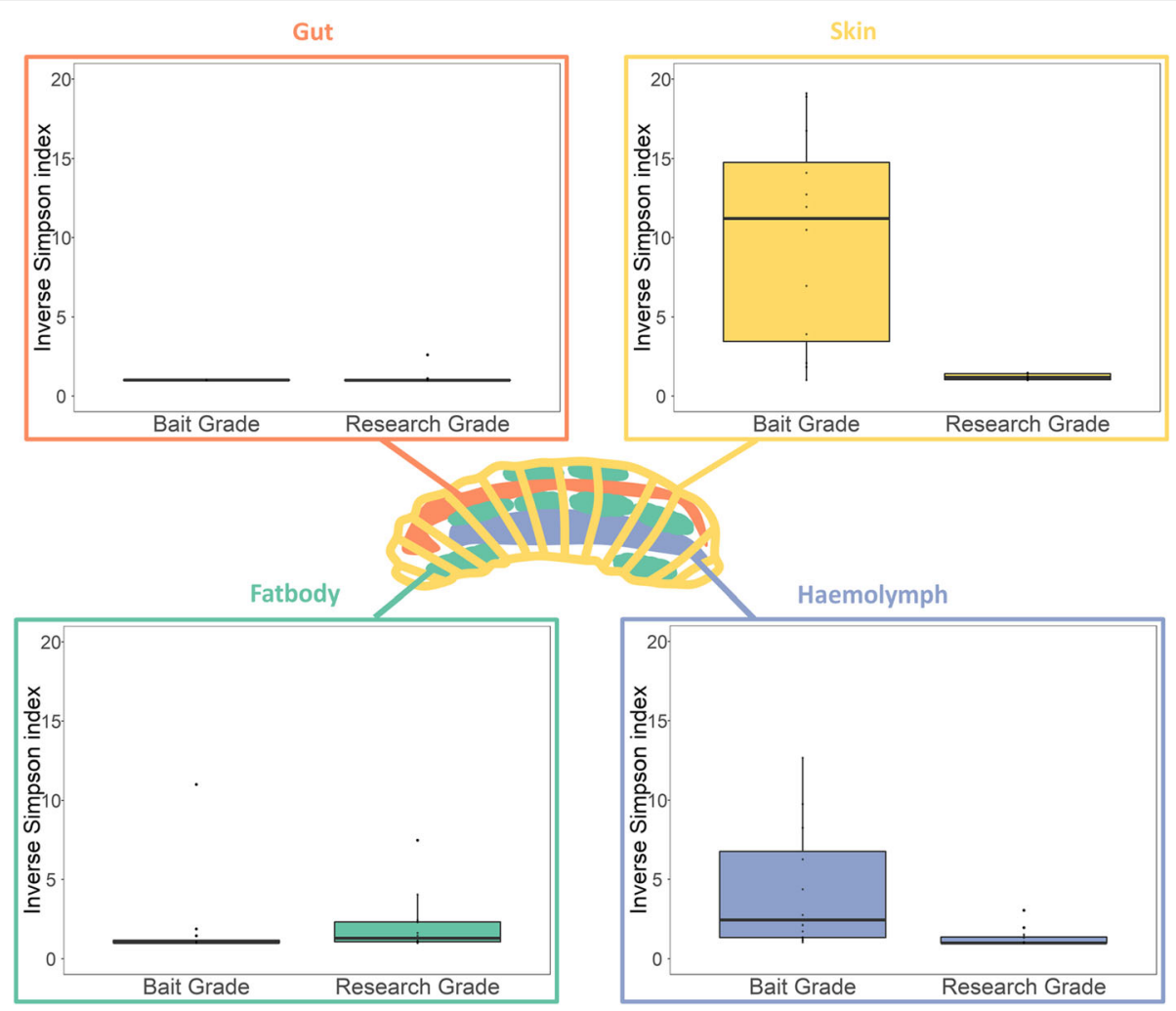

Fig. 2 Diversity measures of four different body sites of bait and research-grade Galleria mellonella larvae. Observed diversity and Inverse Simpson index for fat body $(\mathrm{F})$, faecal $(\mathrm{G})$, haemolymph $(\mathrm{H})$ and skin samples $(\mathrm{S})$ of 12 bait and 12 research-grade larvae

by the same bacterial taxa as research-grade larvae. However, our qPCR did show lower bacterial loads in the baitgrade larvae, possibly resulting from antibiotic administration. It should be taken into account that there is no exact information on hormone or antibiotic administration during rearing available when purchasing bait-grade larvae, while it is guaranteed that the research-grade larvae are grown under very strict rearing conditions. These antibiotics or hormones fed can still be present during your experiment and impact the results. In addition, feeding of antibiotics can enlarge the pool of microbial resistance genes, which can in turn affect experimental outcomes [5].

\section{Conclusions}

Our results corroborate the potential of the Galleria mellonella larvae as a model host system due to the relative simple microbiome present on the different body sites and the high comparability in microbiome between individual larvae. In addition, our results indicate that the microbiome of bait larvae can be compared to the more controlled research grade larvae, which also improves the comparability between different experimental set-ups.

\section{Methods}

Larvae and sample collection

Bait Galleria mellonella larvae were purchased from Anaconda reptiles (Kontich, Belgium) and research-grade Galleria mellonella larvae, grown without antibiotics and hormones, were a gift from $\operatorname{Trularv}^{\mathrm{mm}}$ (BioSystems $^{\mathrm{mm}}$, Devon, U.K.). Upon arrival, the larvae were stored at $4{ }^{\circ} \mathrm{C}$ and used within 7 days. Samples from the skin, faeces, fat body and haemolymph from 12 bait and 12 researchgrade larvae were obtained after one day of individual incubation in the dark at $37^{\circ} \mathrm{C}$.

\section{DNA extraction and bacterial enrichment}

The PowerFecal $^{\bullet}$ DNA isolation kit (with Inhibitor Removal Technology ${ }^{\circ}$ ) was used according to the instructions of the manufacturer.

\section{qPCR}

The isolated DNA samples were diluted 10-fold and used to determine bacterial load by $\mathrm{qPCR}$, using the StepOnePlus real time qPCR system (Applied Biosystems) and $\mathrm{SYBR}^{\circ}$ Green chemistry (PowerUp ${ }^{\mathrm{Tm}} \mathrm{Sybr}^{\circ}$ Green Master Mix, Applied Biosystems). Primers used were 338F (ACTCCTACGGGAGGCAGCAG) and 518R (ATTACCGCGGCTGCTGG) with the followingcycling 

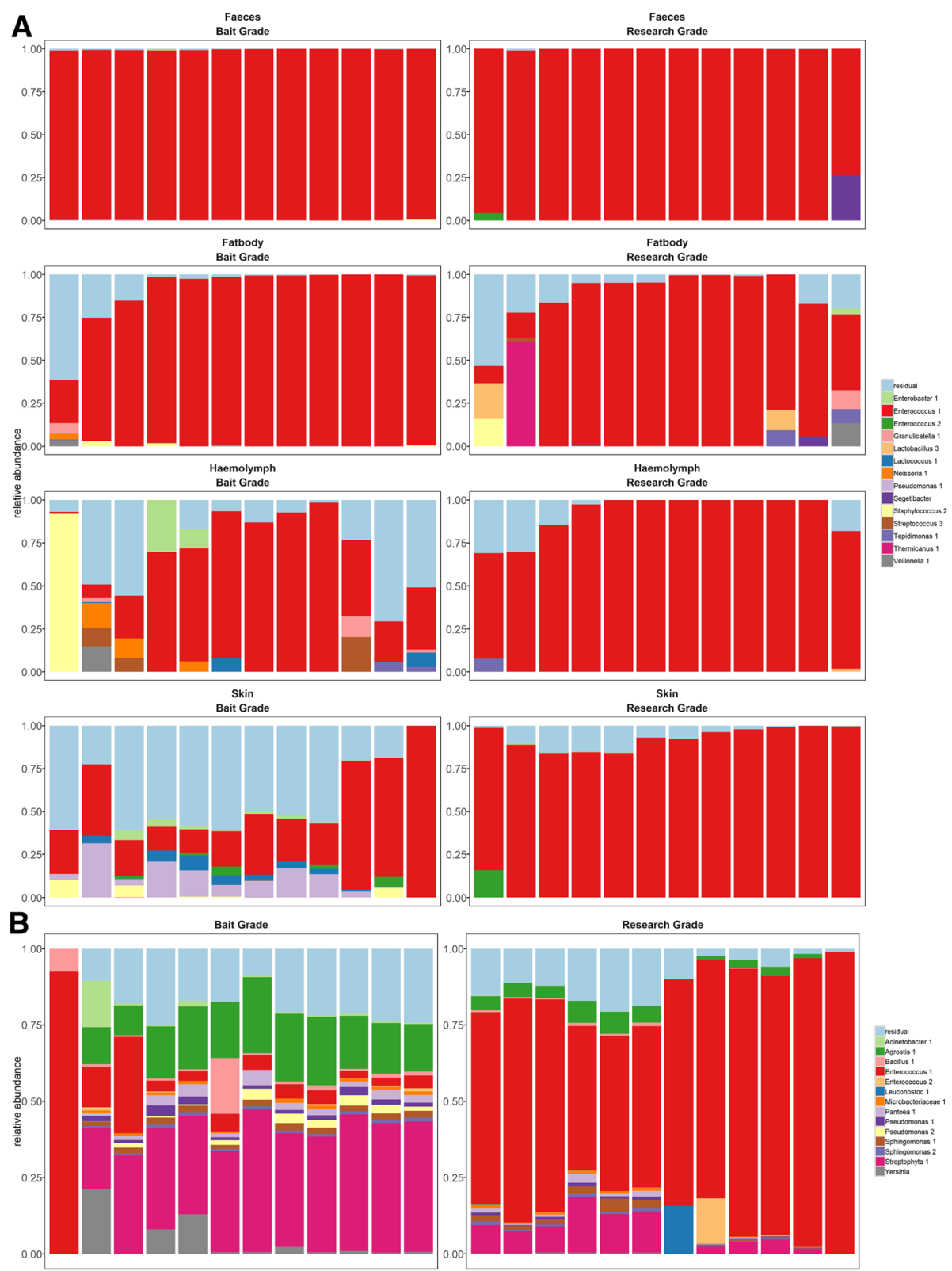

Fig. 3 The microbiome composition on different body sites of G. mellonella larvae. a Comparison of top 14 most abundant amplicon sequence variants (ASVs) in four different body sites (fat body, faecal, haemolymph and skin samples) sampled of 12 bait and 12 research-grade G. mellonella larvae. b Comparison of the top 14 most abundant bacterial and non-bacterial ASVs on the skin. Community profiles are shown for each sample, depicting the relative abundance of the 14 most abundant ASVs. All others ASVs were grouped under residual

program: 3 min at $95^{\circ} \mathrm{C}$; 40 cycles of $1 \mathrm{~min}$ at $95^{\circ} \mathrm{C}, 40 \mathrm{~s}$ at $56^{\circ} \mathrm{C}$ and $40 \mathrm{~s}$ at $72{ }^{\circ} \mathrm{C}[19]$. Based on the difference in $\mathrm{Ct}$-value, an estimation of concentration difference can be made based on the $2^{-\Delta \Delta \mathbf{C t}}$ method.

\section{Illumina MiSeq 165 rRNA amplicon sequencing}

The primers used for Illumina MiSeq sequencing were based on the previously described 515F-806R primers and altered for dual-index paired-end sequencing, as described by Kozich et al. (2013) [20, 21]. Briefly, each
DNA sample was subjected to dual barcoded PCR, amplifying the V4 region of the 16S rRNA gene using Phusion High-Fidelity DNA polymerase (New England Biolabs, USA). PCR products were purified by the Agencourt AMPure XP magnetic bead capture kit (Beckman Coulter, Suarlee, Belgium), and quantified using the Qubit ${ }^{\circ} 3.0$ fluorometer. The library was prepared by pooling all PCR samples in equimolar concentration and loaded onto a $0.8 \%$ agarosegel. The product was purified by gel extraction using the Nucleospin ${ }^{\circ}$ Gel and PCR 
clean-up (Machery-Nagel). The purified library concentration was determined with the $\mathrm{Qubit}^{\circ} 3.0$ fluorometer and diluted to a final concentration of $2 \mathrm{nM}$. The library was denatured with $0.2 \mathrm{~N} \mathrm{NaOH}$ (Illumina), diluted to 6 $\mathrm{pM}$ and spiked with 10\% PhiX control DNA (Illumina). The library was loaded onto the flow cell of the v2 chemistry MiSeq reagent kit (paired-end dual indexing sequencing; $2 \times 251$ bp kit; Illumina, San Diego, California, USA) on the MiSeq Desktop sequencer (M00984, Illumina) at the Centre of Medical Genetics, University of Antwerp, Belgium.

\section{Sequence processing and biostatistical analysis}

Raw sequencing reads were filtered and denoised using the DADA2 (Divisive Amplicon Denoising Algorithm 2) pipeline ( $\mathrm{v}$ 1.1.6), as described in [22]. The DADA2 method is a denoising algorithm that infers the set of most specific biological variants (called amplicon sequence variants (ASVs)) that are not the result of sequencing errors. In short, paired reads were filtered by excluding reads with more than two expected errors and reads that contained undetermined bases. Based on a visual inspection of the quality score profiles, trimming was done by removing first 12 nucleotides on forward and reverse strand. Next, DADA error correction was applied using the error model constructed by alternation of sample inference and error rate estimation until convergence. Forward and reverse reads were then merged into contigs. At this point, chimeras were removed. Taxonomic annotation from the kingdom to the genus level was then assigned to the remaining ASVs, making use of the EzBioCloud 16S rRNA reference database (version mtp1.5, update 2018.05). Finally, ASVs classified as Archaea, Eukarya, chloroplasts or mitochondria were removed. Secondly an alternative analysis was performed containing also non-bacterial ASVs.

The resulting ASV table was imported and analysed in $\mathrm{R}$, using the in-house developed package tidyamplicons (www.github.com/swittouck/tidyamplicons), ggplot2 (v 2.1.0) [23], and the vegan package (v 2.3-5) [24]. Quality control was performed as described in the Result section. Observed ASV-richness and the inverse Simpson index calculated on the non-normalized read count data were used as alpha-diversity indices. The relative abundances of the top 14 ASVs were plotted to assess the bacterial community composition.

\section{Abbreviations}

ASV: Amplicon sequence variant; DADA2: Divisive Amplicon Denoising Algorithm 2; DNA: Deoxyribonucleic acid; MRS: De Man Rogosa Sharpe; PCR: Polymerase chain reaction; rRNA: Ribosomal ribonucleic acid

\section{Acknowledgements}

The authors want to thank the entire research group ENdEMIC of the University of Antwerp, especially Ines Tuyaerts for loading the MiSeq library, and Freya Cools and Paul Cos from the research group LMPH of the
University of Antwerp. All computational work was performed on Lamborgenei.

\section{Authors' contributions}

CNA, WVB and SL designed experiments, performed wet-lab extractions, microbiome analysis (in silico) and drafted the manuscript. IDB helped with preparation of the $16 \mathrm{~S}$ amplicon sequencing. SW helped with the analysis (in silico) of the 165 amplicon sequencing (denoising). All authors read and approved the final manuscript.

\section{Funding}

CNA (FWO-SB fellowship 1S $03516 \mathrm{~N}$ ), WVB (Dehousse fellowship from University of Antwerp, IDB (FWO-SB fellowship $1 S 17916$ N) and SW (FWOAsp fellowship 11A0618N).

\section{Availability of data and materials}

Sequencing data are available at the European Nucleotide Archive with the accession number PRJEB31807 (https://www.ebi.ac.uk/ena/data/view/PRJEB31 807). Scripts used for generating the figures and performing quality control are available at https://github.com/LebeerLab/Galleria.

Ethics approval and consent to participate

Not applicable.

\section{Consent for publication}

Not applicable.

\section{Competing interests}

The authors declare that they have no competing interests.

Received: 16 May 2019 Accepted: 21 July 2019

Published online: 03 September 2019

\section{References}

1. Tsai CJ-Y, Loh JMS, Proft T. Galleria mellonella infection models for the study of bacterial diseases and for antimicrobial drug testing. Virulence. 2016;7(3): 214-29.

2. Kavanagh K, Fallon JP. Galleria mellonella larvae as models for studying fungal virulence. Fungal Biol Rev. 2010;24(1-2):79-83.

3. Champion OL, Wagley S, Titball RW. Galleria mellonella as a model host for microbiological and toxin research. Virulence. 2016;7(7):840-5.

4. Johnston PR, Rolff J. Host and symbiont jointly control gut microbiota during complete metamorphosis. Schneider DS, editor. PLoS Pathog. 2015; 11(11):e1005246.

5. Ignasiak K, Maxwell A. Oxytetracycline reduces the diversity of tetracyclineresistance genes in the galleria mellonella gut microbiome. BMC Microbiol. 2018;18(1):228.

6. Wollenberg AC, Jagdish $T$, Slough $G$, Hoinville ME, Wollenberg MS. Death becomes them: bacterial community dynamics and stilbene antibiotic production in cadavers of galleria mellonella killed by Heterorhabditis and Photorhabdus spp. Appl Environ Microbiol. 2016;82(19):5824-37 Elliot MA, editor.

7. Polenogova OV, Kabilov MR, Tyurin MV, Rotskaya UN, Krivopalov AV, Morozova W, et al. Parasitoid envenomation alters the galleria mellonella midgut microbiota and immunity, thereby promoting fungal infection. Sci Rep. 2019;9(1):4012.

8. Blum JE, Fischer CN, Miles J, Handelsman J. Frequent replenishment sustains the beneficial microbiome of Drosophila melanogaster. MBio. 2013;4(6): e00860-13-e00860-13.

9. Ridley EV, Wong AC-N, Westmiller S, Douglas AE. Impact of the resident microbiota on the nutritional phenotype of Drosophila melanogaster. PLoS One. 2012;7(5):e36765 Leulier F, editor.

10. Bates JM, Mittge E, Kuhlman J, Baden KN, Cheesman SE, Guillemin K. Distinct signals from the microbiota promote different aspects of zebrafish gut differentiation. Dev Biol. 2006;297(2):374-86

11. Jervis-Bardy J, Leong LEX, Marri S, Smith RJ, Choo JM, Smith-Vaughan HC, et al. Deriving accurate microbiota profiles from human samples with low bacterial content through post-sequencing processing of Illumina MiSeq data. Microbiome. 2015;3(1):19. 
12. Salter SJ, Cox MJ, Turek EM, Calus ST, Cookson WO, Moffatt MF, et al. Reagent and laboratory contamination can critically impact sequence-based microbiome analyses. BMC Biol. 2014;12(1):87.

13. Ratcliffe NA, Walters JB. Studies on the in vivo cellular reactions of insects: clearance of pathogenic and non-pathogenic bacteria in galleria mellonella larvae. J Insect Physiol. 1983;29(5):407-15.

14. Kim O-S, Cho Y-J, Lee K, Yoon S-H, Kim M, Na H, et al. Introducing EzTaxone: a prokaryotic $16 \mathrm{~S}$ rRNA gene sequence database with phylotypes that represent uncultured species. Int J Syst Evol Microbiol. 2012;62(Pt 3):716-21.

15. Lebreton F, Willems RJL, Gilmore MS. Enterococcus diversity, origins in nature, and gut colonization [internet]. Enterococci: from commensals to leading causes of drug resistant infection. Massachusetts Eye and Ear Infirmary; 2014. pmid: 24649513.

16. Milani C, Duranti S, Bottacini F, Casey E, Turroni F, Mahony J, et al. The first microbial colonizers of the human gut: composition, activities, and health implications of the infant gut microbiota. Microbiol Mol Biol Rev. 2017;81(4).

17. Layton BA, Walters SP, Lam LH, Boehm AB. Enterococcus species distribution among human and animal hosts using multiplex PCR. J Appl Microbiol. 2010;109(2):539-47.

18. Kim K, Lee G, Thanh HD, Kim J-H, Konkit M, Yoon S, et al. Exopolysaccharide from Lactobacillus plantarum LRCC5310 offers protection against rotavirusinduced diarrhea and regulates inflammatory response. J Dairy Sci. 2018; 101(7):5702-12.

19. Van Herreweghen F, De Paepe K, Roume H, Kerckhof F-M, Van de Wiele T. Mucin degradation niche as a driver of microbiome composition and Akkermansia muciniphila abundance in a dynamic gut model is donor independent. FEMS Microbiol Ecol. 2018;94(12):fiy186 pmid: 30239657.

20. Caporaso JG, Lauber CL, Walters WA, Berg-lyons D, Lozupone CA, Turnbaugh PJ, et al. Global patterns of $16 \mathrm{~S}$ rRNA diversity at a depth of millions of sequences per sample. Proc Natl Acad Sci U S A. 2010;108(1).

21. Kozich JJ, Westcott SL, Baxter NT, Highlander SK, Schloss PD. Development of a dual-index sequencing strategy and curation pipeline for analyzing amplicon sequence data on the MiSeq Illumina sequencing platform. Appl Environ Microbiol. 2013;79(17):5112-20 pmid: 23793624.

22. Callahan BJ, Mcmurdie PJ, Rosen MJ, Han AW, Johnson AJ, Holmes SP. DADA2: high-resolution sample inference from Illumina amplicon data. Nat Methods. 2016:13(7):581 pmid: 27214047.

23. Ginestet C. ggplot2: elegant graphics for data analysis. J R Stat Soc Ser A (Statistics Soc). 2011;174(1):245-6 pmid: 387981403.

24. Oksanen J, Blanchet F, Kindt R, Legendre P, O'Hara R. Vegan: community ecology package. R Packag 23-3. 2016; pmid: 17666792.

\section{Publisher's Note}

Springer Nature remains neutral with regard to jurisdictional claims in published maps and institutional affiliations.

Ready to submit your research? Choose BMC and benefit from:

- fast, convenient online submission

- thorough peer review by experienced researchers in your field

- rapid publication on acceptance

- support for research data, including large and complex data types

- gold Open Access which fosters wider collaboration and increased citations

- maximum visibility for your research: over $100 \mathrm{M}$ website views per year

At $\mathrm{BMC}$, research is always in progress.

Learn more biomedcentral.com/submissions 